\title{
Neural-Network-Controlled Single-Phase UPS Inverters with Improved Transient Response and Adaptability to Various Loads
}

\author{
Xiao Sun*, Dehong Xu**, Frank H.F.Leung*, Yousheng Wang** and Yim-Shu Lee* \\ * Department of Electronic and Information Engineering \\ The Hong Kong Polytechnic University \\ Hung Hom, Kowloon, Hong Kong \\ ** Department of Electrical Engineering \\ Zhejiang University \\ Hangzhou 310027, China
}

\begin{abstract}
This paper proposes a neural-network control scheme for the inverters of Uninterruptible Power Supplies (UPS) to improve their transient response and adaptability to various loads. Two simulation models are built to obtain example patterns for training the neural network. One is a multiple-feedback-loop controller for linear loads, and the other is an idealized load-current-feedback controller specially designed for nonlinear loads. The latter controller has a built-in reference load model, and the load current is forced to track this reference. Example patterns under various loading conditions are used in the off-line training of a selected neural network, which is made as simple as possible to reduce the calculation time. When the training is completed, the neural network is used to control the UPS inverter on-line. The development of example patterns and training of the neural network are done using MATLAB and SIMULINK, and the verification of results is done using PSpice. It is found that the proposed neural-networkcontrolled inverter can provide good sinusoidal output voltage with low Total Harmonic Distortion (THD) under various loading conditions, and good transient responses when the load changes.
\end{abstract}

\section{INTRODUCTION}

When utility power fails, Uninterruptible Power Supplies (UPS) should continue to provide electric power to critical systems such as communication systems, computer systems, and life support systems. The UPS inverter is required to produce constant sinusoidal output voltage with minimum Total Harmonic Distortion (THD) for a wide load range: from resistive to inductive or capacitive, and from linear to nonlinear. At the same time, fast transient response, good stability and high reliability are also required.

In a typical UPS inverter there is a Pulse-Width Modulation (PWM) converter and a second-order LC output filter. Various controllers were used to achieve regulation. The controllers can be classified into two groups: analogbased and digital-based. Most of the analog-based controllers were designed based on linearized model and traditional frequency-domain analysis. Some multiple-feedback-loop control strategies were proposed in [1, 2]. Such schemes sense the current in the inductor (or capacitor) of the LC filter as the control signal of an inner feedback loop. An outer voltage feedback loop is incorporated to ensure a sinusoidal output voltage. Owing to the introduction of the inner current feedback loop, the output impedance of the inverter filter is decreased and the dynamic stiffness enhanced. The controlled inverter can produce a satisfactory sinusoidal output voltage within a certain load range. However, a uniform performance cannot be obtained under extreme loading conditions, and THD increases significantly when there is a nonlinear load. In addition, such schemes are analog-based, which suffer from all the drawbacks of analog circuits, such as limited accuracy, uncompensated thermal drift, and need of recalibration.

Artificial Neural Network (ANN) techniques have grown rapidly in recent years. An ANN is an interconnection of a number of artificial neurons that simulates a real biological brain system, which can compute very fast in a parallel and distributed manner. It also has the ability to approximate an arbitrary nonlinear mapping. Compared with other digital algorithms, although the training process of an ANN is timeconsuming and complex, the actual real-time computation from input to output is fast and simple [3]. ANN has already been employed in various control and signal processing applications in power electronics and drives [4][5]. In the control of DC-AC inverters, ANN has been introduced in the current control of inverters for $\mathrm{AC}$ motor drives, where the ANN receives a phase-current error and generates PWM logic signals to drive the inverter switches [6]-[9]. The neural network has been used in the harmonic elimination of PWM converters where the ANN replaced a large and memorydemanding look-up table to generate the switching angles of a PWM converter for a given modulation index [10][11]. The neural network was also introduced into UPS inverter control [12]. In this method, the neural network is trained off-line, and the trained network can be employed for on-line control. The inputs of the neural network are time, present output voltage and last sampled output voltage. However, the ANN used may not have enough information to ensure a sinusoidal output voltage under extreme loading conditions.

This paper proposes a neural network control scheme for UPS inverters, which learns the control law from

This project was supported by The Hong Kong Polytechnic University and Zhejiang University. 
representative example patterns obtained from two simulation models. One model is a multiple-feedback-loop controller for linear loads, which has been widely used in the control of power converters, the other is an idealized load-currentfeedback controller specially designed for nonlinear loads. The latter controller has a built-in reference load model, and the actual load current is forced to track this reference. This idealized model is not easy to implement experimentally since the load model cannot be obtained beforehand. A selected neural network is trained off-line with the patterns obtained from simulations. The inputs to the neural network are the present capacitor current, previously sampled capacitor current, present load current, present output voltage, and the error between the reference voltage and the output voltage. The output of the neural network is a part of modulation signal to the PWM generator. The whole modulation signal consists of the output of the neural network and a sinusoidal voltage reference. When the training is completed, this neural network is used to control the inverter on-line. Development of the example patterns and training of the neural network are done using MATLAB and SIMULINK, and performance verifications are done using PSpice. Simulation results have proven that the proposed neural-network-controlled inverter can provide a good sinusoidal output voltage with low THD under various loading conditions, and good transient responses when the load changes.

\section{ARCHITECTURE OF UPS INVERTER}

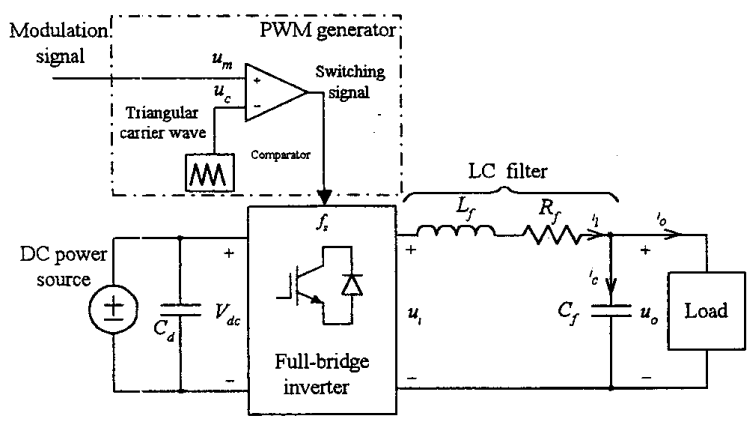

Fig.1. Single-phase UPS inverter system

A UPS inverter typically consists of a DC power source, a full-bridge (or half bridge) PWM inverter and an LC filter, as shown in Fig. 1. The full-bridge inverter, which is the core of the system, chops the DC input into a series of PWM pulses according to the modulation signal $u_{m}$. The function of the second-order LC filter is to remove the high frequency components of the chopped output voltage $u_{i}$. $R_{f}$ represents the resistance of the filter inductor. The effective series resistance (ESR) of the filter capacitor is ignored since it only has a small effect within the frequency range concerned. The $\mathrm{DC}$ power source is considered as an ideal constant-voltage supply. The load shown in Fig.1 can be of any type: resistive, inductive, capacitive, or nonlinear.

Because the switching frequency (here is $20 \mathrm{KHz}$ ) is usually several orders higher than the fundamental frequency of the AC output, the dynamics of the PWM inverter can be ignored. Thus, the UPS inverter can be modeled as a simple proportional gain block. Fig. 2 shows a linear model of the inverter system (PWM inverter plus the output filter and the load), in which the proportional gain of the inverter $K$ is equal to $V_{d c} / V_{c}\left(V_{d c}\right.$ is the voltage of the DC power source and $V_{c}$ is the peak voltage of the triangular carrier wave).

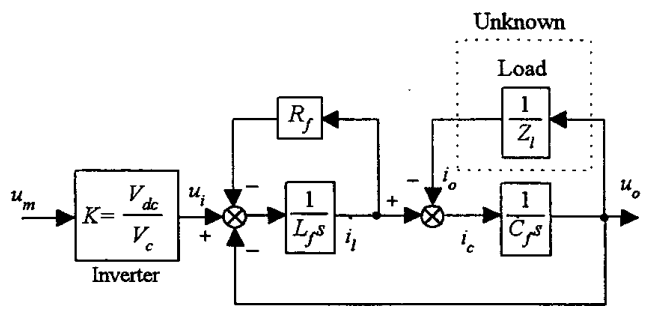

Fig.2. Linear model of UPS inverter

\section{PROPOSED NEURAL NETWORK CONTROL OF INVERTER}

Artificial Neural networks are interconnections of artificial neurons that tend to emulate the human brain. One structure of the artificial neuron is shown in Fig.3. Here the individual element inputs, $x_{1}, x_{2}, x_{3}, \ldots x_{n}$, are multiplied by weights, $W_{l j}$, $W_{2 j}, W_{3 j}, \ldots W_{n j}$, and the weighted values are fed to the summing node. The neuron has a bias $b$, which is summed with the weighted inputs to form the net input $R_{j} . R_{j}$ is then used as the argument of the transfer function $f$. Therefore, the output of the neuron can be written as:

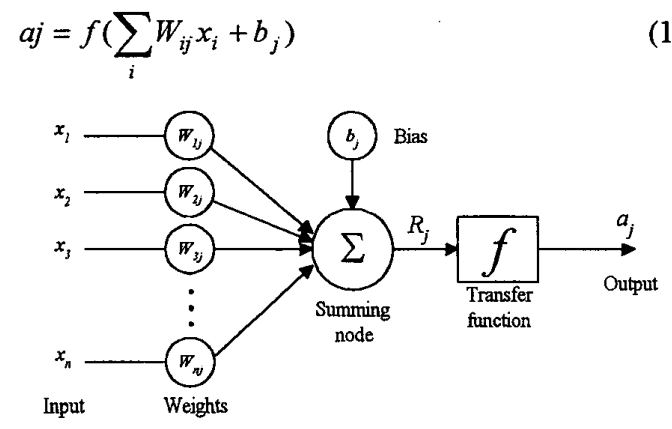

Fig.3. Structure of an artificial neuron

The transfer function can be of threshold type, sigmoid type, linear type etc. A neural network can be classified into feedforward or feedback type, depending on the interconnection of the neurons. At present, the vast majority of applications use the feedforward architecture, which will also be used in this paper. Fig.4 shows the structure of a feedforward multi-layer network that has one hidden layer. The 
circles represent neurons, which have the structure as shown in Fig.3. A weight-adjustment feature is included for implementing the back propagation. There can be more than one hidden layer. The number of hidden layers and the number of neurons on each layer depend on the complexity of the problem being solved and the desired accuracy. The back propagation algorithm is most commonly used for the training of feedforward neural networks. It updates the network weights and biases in the direction that the performance function, usually the sum of square errors, decreases most rapidly. The training is usually done by an off-line computer simulation program using a large number of example patterns. The example patterns can be obtained from analysis, simulation or directly from experiments if the model is totally unknown.

To realize the proposed neural network control scheme on inverters, we have to present a large number of example patterns obtained under various loading conditions, and train the neural network properly to implement the control law. Two controllers, one for linear loads, the other for nonlinear loads, are built and simulated using MATLAB and SIMULINK.

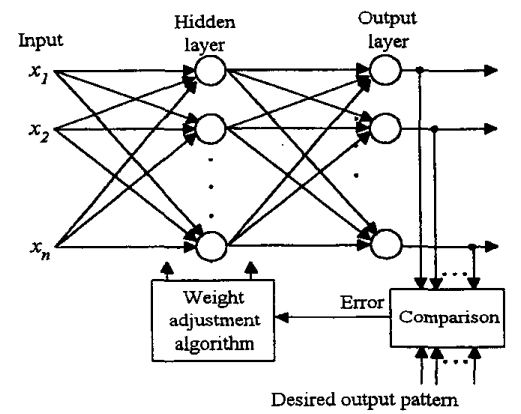

Fig.4. Structure of a two layer feedforward neural network showing the back propagation training

\section{A. Obtaining Example Patterns under Linear Loading Conditions}

Under linear load conditions (resistive, capacitive, or inductive), a multiple-feedback-loop control scheme can give good performance $[1,2]$. The control scheme for linear loads is shown in Fig.5. Different from the strategy proposed in [1, 2], there is a feedforward signal from the reference voltage (shown at the top of Fig.5), which is found to have advantages of reducing steady-state error and providing a high tracking accuracy to the reference. Although it may cause large overshoot in the dynamic response, the drawback can be overcome by optimizing the parameters of the voltage feedback loop. The modulation signal to the PWM generator consists of two components, one is a sinusoidal feedforward signal, and the other is a compensation signal (as marked in the middle of Fig.5) produced by feedback loops. In this way, the neural network can be trained to generate only the "compensation signal" as its desired output. This will result in a more effective training and a better control performance.

An inverter system with multiple-feedback-loop control scheme is modeled using MATLAB and SIMULINK to obtain the example patterns under linear loading conditions. The fullbridge inverter is described by the following equation in MATLAB:

$u_{i}=\left\{\begin{array}{cc}V_{d c} & \left(u_{m} \geq u_{c}\right) \\ -V_{d c} & \left(u_{m}<u_{c}\right)\end{array}\right.$

where $u_{m}$ is the instantaneous voltage of the modulating signal and $u_{c}$ is the instantaneous voltage of the triangular carrier wave in the PWM.

It should be noted that a fixed parameter controller cannot be good for all kinds of linear loads. Each load is associated with a set of optimal parameters. We design the parameters for each load using frequency-domain analysis to ensure enough stability margin and small steady-state error, and fine-tune the parameters in the simulations for good transient responses. The compensation signal as shown in Fig. 5 is collected as the desired output of the neural network. The output voltage and output currents (including load current, capacitor current) of the inverter are collected as the inputs to the neural network. Dozens of example patterns are obtained from the simulation results under different load conditions and controller parameters for training purposes.

\section{B. Obtaining Example Patterns under Nonlinear Loading Conditions}

Many electrical loads nowadays are nonlinear. It is therefore essential to maintain the performance of a UPS inverter under nonlinear loading conditions. The nonlinear load in this paper is chosen to be a full-wave diode bridge rectifier with an output filter. The input current of the nonlinear load, namely the output current of the inverter, is usually nonsinusoidal with a rather large $d i / d t$. The multiple-feedbackloop control scheme used under the linear loading conditions cannot perform satisfactorily. Thus, we should find another control scheme especially for nonlinear loads in order to obtain example patterns.

If the load current of the inverter can be predicted, we can design a controller to keep track of the output current. Following the control scheme used under the linear loading conditions, we change the inner capacitor-current loop to a load-current loop whose reference is obtained from an idealized load-current feedback control scheme (as shown in Fig.6), where a sinusoidal voltage is fed to a nonlinear load model to obtain a load-current reference. The actual load current is compared with this reference, and the error signal is used as the controller input. Although such scheme is not easy to implement practically, we can perform simulations to obtain example patterns for training the neural network.

A model of such an idealized load-current feedback control scheme is built using MATLAB and SIMULINK. The PWM full-bridge inverter is modeled in the way as mentioned in the last subsection. The core of modeling a full-wave diode bridge 


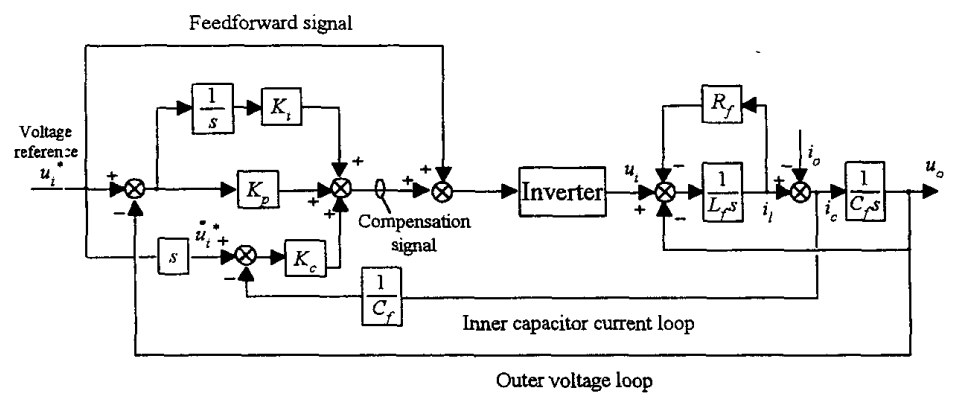

Fig.5. Multiple-feedback-loop control scheme to obtain example patterns for linear loads

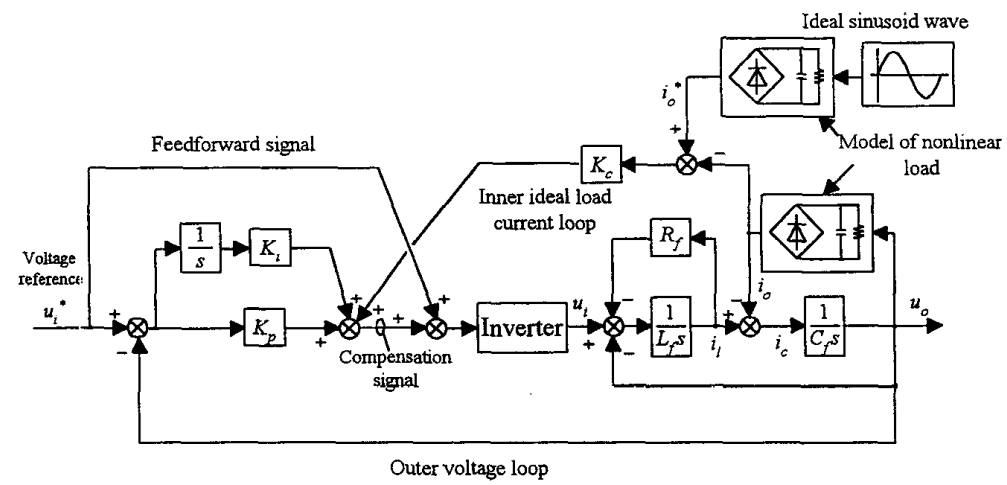

Fig.6. Idealized load-current feedback control scheme to obtain example patterns for nonlinear loads

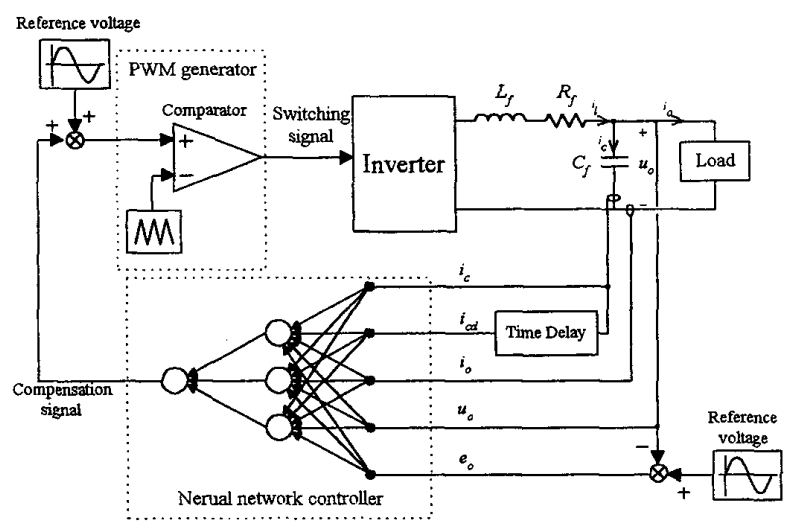

Fig.7. Proposed neural network control scheme for UPS inverter

rectifier in MATLAB is the model of the power diode. We describe a diode using the following equation:

$i_{d}=\left\{\begin{array}{cc}0 & u_{d}<0.7 \\ \left(u_{d}-0.7\right) / 0.1 & u_{d} \geq 0.7\end{array}\right.$

where $u_{d}$ is the instantaneous forward voltage across the diode and $i_{d}$ is the instantaneous forward current in the diode.

The parameters of controller are determined from the simulations to produce an output with a low THD and a small enough steady-state error. Similar to the linear loads, dozens of example patterns based on scheme are obtained under various output-filter capacitors and load resistances for training purposes.

\section{Structure and training of Neural Network}

All the example training patterns obtained from the simulations are put together to form a database that contains the information about the control law. A proper neural network 
is then used to learn the control law. The neural network should be as simple as possible to reduce the calculation time. Fig. 7 shows the proposed neural-network-controlled inverter. The neural network is of a 5-3-1 structure (five inputs, three nodes in hidden layer and one output). The inputs are present capacitor current, last sampled capacitor current, present load current, present output voltage, and the error between the reference voltage and the output voltage The sampling frequency is set to be $10 \mathrm{KHz}$. This structure is the result of many repeated trials.

To achieve a trained neural network that can perform well for both linear and nonlinear loads, it is found from many trials that the pattern database should consist of about twothird of patterns for linear loads and about one-third of patterns for nonlinear loads.

The training of the neural network is done off-line using MATLAB with Neural Network Toolbox. The LevenbergMarquardt algorithm is used in the training, which has a fast convergence rate. The neural network is trained repeatedly with randomly selected data from the pattern database. After the training is completed, the weights and biases are downloaded to the neural-network controller to control the inverter on-line.

\section{SIMULATION}

To verify the performance of the proposed neural network control scheme, we simulate the neural network controlled inverter system using PSpice, which has accurate models of switching components and diodes. The weights and biases from MATLAB simulations are put into the model in PSpice. Table 1 lists the parameters of the inverter used in the simulation.

\begin{tabular}{lcc}
\multicolumn{3}{c}{ TABLE 1 INVERTER PARAMETERS } \\
\hline \multicolumn{1}{c}{ Parameter } & Value & Units \\
\hline Switching frequency, $f_{s}$ & 20 & $\mathrm{KHz}$ \\
Sampling frequency, $f_{s a}$ & 10 & $\mathrm{KHz}$ \\
Nominal DC source voltage, $V_{d c}$ & 420 & $\mathrm{~V}$ \\
Rated Output Voltage & 220 & $\mathrm{~V}_{\mathrm{mms}}$ \\
Rated Output Frequency & 50 & $\mathrm{~Hz}$ \\
Rated Output Current & 11 & $\mathrm{~A}$ ms \\
Rated output Power & 2.4 & $\mathrm{kVA}$ \\
Rated output impedance & 20 & $\Omega$ \\
Filter Inductor, $L_{f}$ & 250 & $\mu \mathrm{H}$ \\
Inductor Resistance, $R_{f}$ & 0.2 & $\Omega$ \\
Filter Capacitor, $C_{f}$ & 33 & $\mu \mathrm{F}$ \\
\hline
\end{tabular}

The performance and transient responses of the proposed neural network controlled inverter are investigated under various load conditions. Fig.8 shows the simulation results of the output voltage and current of the inverter when the load changes from no load to $20 \Omega$ resistive. Fig. 9 shows the simulation result when the load changes from no load to $20 \Omega$ capacitive, with power factor $(\mathrm{PF})=0.6$. Fig.10shows the simulation result when the load changes from no load to $20 \Omega$ inductive, also with $\mathrm{PF}=0.6$. Note that the output voltage recovers very quickly in all cases. Fig. 11 shows the output voltage and current of the inverter for a nonlinear load consisting of a full-wave diode bridge rectifier followed by a $1000 \mu \mathrm{F}$ capacitor in parallel with a $20 \Omega$ resistor. Note that although the current has high spikes, the voltage waveform is distorted only slightly. Table 2 lists the THD of the output voltage under various loading conditions. The simulations show that the proposed neural network controlled inverter can achieve a low THD.

\section{CONCLUSION}

A neural network control scheme for UPS inverter has been presented in this paper. First, the methods for obtaining the example patterns are introduced. Two simulation models are built to obtain example patterns for linear and nonlinear loads respectively. One is a multiple-feedback-loop controller for linear loads; and the other is an idealized load-current feedback controller specially designed for nonlinear loads. Then a neural network is selected and trained using example patterns to formulate the control law. Simulation results show that the proposed neural-network-controlled inverter can provide good sinusoidal output voltage with low THD under various loading conditions, it also has good transient responses.

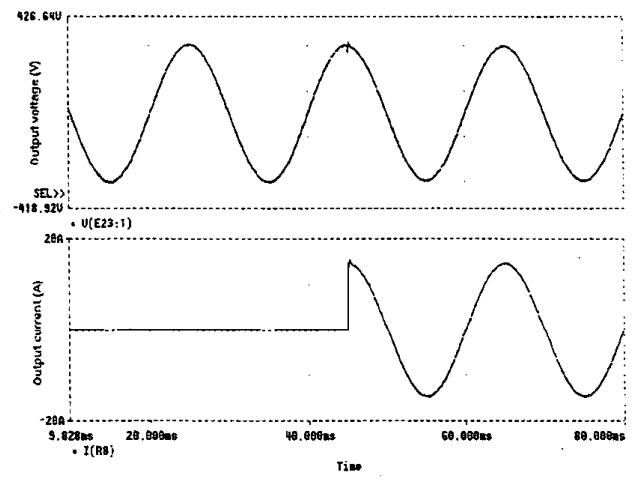

Fig.8 Simulation result when the load changes from open circuit to $20 \Omega$ resistive

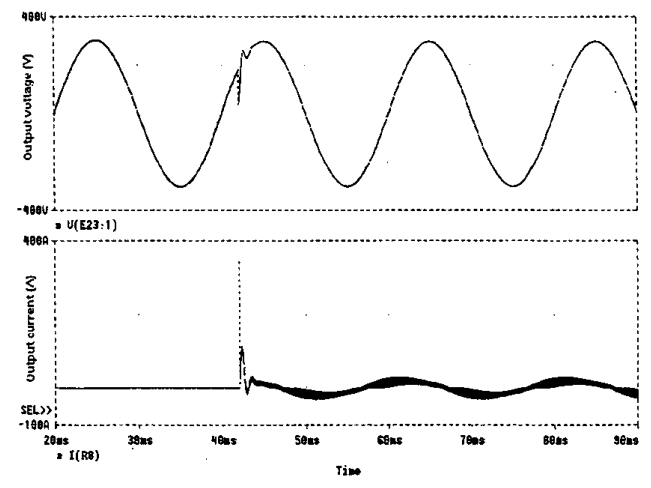

Fig.9 Simulation result when the load changes from open circuit to $20 \Omega$ capacitive with leading $\mathrm{PF}=0.6$ 


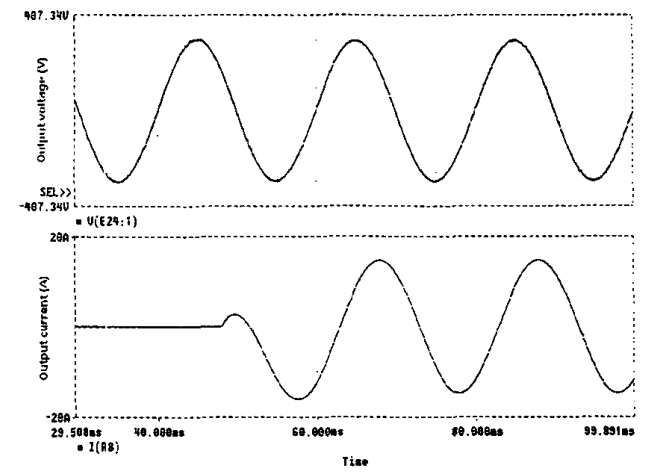

Fig.10 Simulation result when the load changes from open circuit to $20 \Omega$ incuctive with lagging $P F=0.6$

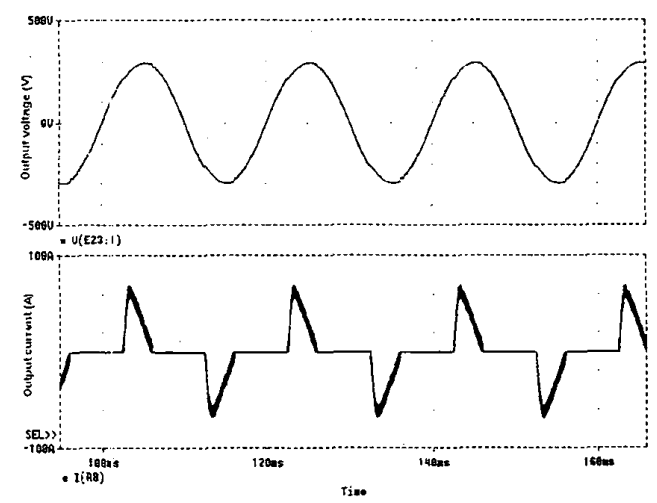

Fig.11 Simulation result for a full-wave diode bridge rectifier load $(1000 \mu \mathrm{F} 20 \Omega)$

TABLE 2 THD OF OUTPUT VOLTAGE UNDER VARIOUS LOADING CONDITIONS

\begin{tabular}{c|c|c|c|c}
\hline \multirow{2}{*}{$\begin{array}{c}\text { Loading } \\
\text { condition; }\end{array}$} & \multirow{2}{*}{ No load } & \multicolumn{4}{|c}{ Resistive load } \\
\cline { 2 - 5 } & & $5 \Omega$ & $10 \Omega$ & $20 \Omega$ \\
\hline THD (\%) & 0.38 & 0.45 & 0.43 & 0.43 \\
\hline \multirow{2}{*}{$\begin{array}{c}\text { Loading } \\
\text { conditions }\end{array}$} & \multicolumn{4}{|c}{$20 \Omega$ capacitive loads } \\
\cline { 2 - 5 } & $0.6 \mathrm{PF} *$ & $0.7 \mathrm{PF}$ & $0.8 \mathrm{PF}$ & $0.9 \mathrm{PF}$ \\
\hline THD (\%) & 0.26 & 0.29 & 0.31 & 0.30 \\
\hline \multirow{2}{*}{$\begin{array}{c}\text { Loading } \\
\text { conditions }\end{array}$} & $0.6 \mathrm{PF}$ & $0.7 \mathrm{PF}$ & $0.8 \mathrm{PF}$ & $0.9 \mathrm{PF}$ \\
\cline { 2 - 5 } THD (\%) & 0.54 & 0.48 & 0.48 & 0.48 \\
\hline \multirow{2}{*}{$\begin{array}{c}\text { Loading } \\
\text { conditions }\end{array}$} & $500 \mu \mathrm{F}$ & $1000 \mu \mathrm{F}$ & $2000 \mu \mathrm{F}$ & $3300 \mu \mathrm{F}$ \\
\cline { 2 - 5 } & $20 \Omega$ & $20 \Omega$ & $20 \Omega$ & $20 \Omega$ \\
\hline THD (\%) & 1.61 & 1.69 & 1.79 & 1.96 \\
\hline * PF - Power Factor &
\end{tabular}

\section{REFERENCES}

[1] N. M. Abdel-Rahim, J. E: Quaicoe, "Analysis and design of a multiple feedback loop control strategy for single-phase voltage-source UPS inverters", IEEE
Trans. Power Electronics, vol.11, no.4, pp.532-541, 1996

[2] M. J. Ryan, W. E. Brumsickle, and R. D. Lorenz, "Control topology options for single-phase UPS inverter", IEEE Trans. Ind. Applicat. , vol.33, no.2, pp.493-501, 1997

[3] K. J. Hunt, D. Sbarbaro, R. Zbikowski and P. J. Gawthrop, "Neural networks for control system - a survey" Automatica, vol.28, no.6, pp.1083-1112, 1992

[4] B. K. Bose, "Expert system, fuzzy logic, and neural network applications in power electronics and motion control", Proceeding of the IEEE, vol.82, no.8, pp.13031323, 1994

[5] B. R. Lin, "Analysis of neural and fuzzy-power electronic control", IEE Proc.- Sci. Meas. Technol., Vol.144, No.1, pp.25-33, 1997

[6] Y. Ito, T. Furuhashi, S. Okuma, Y. uchikawa, "A digital current controller for a PWM inverter using a neural network and its stability", ", IEEE Power Electronics Specialists Conf. , pp.219-224, 1990

[7] M. R. Buhl, R. D. Lorenz, "Design and implementation of neural networks for digital current regulation of inverter drives", in IEEE/LAS Annu. Meet. Conf. Rec., pp415-423, 1991

[8] B. R. Lin, R. G. Hoft, "Power electronics inverter control with neural networks", in IEEE-APEC Conf. Rec., pp.128-134, 1993

[9] M. P. Kazmierkowski, D. Sobczuk, "Improved neural network current regulator for VS-PWM inverters", in IEEE-IECON'94, Conf, Rec., pp.1237-1241, 1994

[10] A. M. Trzynadlowski, S. Legowski, "Application of neural networks to the optimal control of three-phase voltage-controlled inverters", IEEE Trans. Power Electronics, vol.9, no.4, pp.397-404, 1994

[11] D. Daniolos, M. K. Darwish and P. Mehta, "Optimized PWM inverter control using artificial neural networks", Electronics Letters, vol.31, no.20, pp.1739-1740, 1995

[12] R. C. Hwang, T. J. Liang, J. W. Chen, "Neural networks controlled PWM inverter', IEEE-INTELEC'97 Conf, Rec., pp.201-206, 1997 